\title{
EFFECT OF AGEING AND OSTEOARTHRITIS OF KNEE OVER TEMPOROSPASTIAL PARAMETERS OF GAIT: A SYSTEMIC REVIEW
}

\author{
Ranveer Kumar Mahato ${ }^{* 1}$, Shreya Patel ${ }^{2}$, Neeta J Vyas ${ }^{3}$.
}

${ }^{* 1}$ Ph.D. Scholar, Gujarat University, Ahmedabad, Principal, Pioneer Physiotherapy College, Ajwa-Nimeta Road, Sayajipura, Vadodara, Gujarat, India.

${ }^{2}$ Lecturer, Pioneer Physiotherapy College, Ajwa-Nimeta Road, Sayajipura, Vadodara, Gujarat, India.

${ }^{3}$ Principal, IKDRC-ITS College of Physiotherapy, Medicity, Ahmedabad, Gujarat, India.

\section{ABSTRACT}

Background: Health care provision is facing significant burden due to osteoarthritis, which is the most common condition affecting synovial joints. Osteoarthritis of the knee causes substantial pain and disability, especially in the elderly.

Purpose of the study: Our objective is to incorporate the effect of ageing and osteoarthritis of knee over temporospatial parameters of gait, based on a systematic review of the evidence from various articles.

Method: Systemic literature search using PubMed, CINAHL, EBSCO and Willes library databases done. Articles following criteria of OA knee, gait analysis and ageing were selected.

Result: Among the 272 articles identified by the literature search, 59 articles reporting one of filter criteria were selected. Three additional articles were found by manual search. After reviewing the full-texts, another 29 reports were excluded because preselected 3D kinematic data were not available. Thus, the final analysis included 30 full-length reports (19 knee OA studies, 11 OA studies including ageing individuals) studying 781 knee OA patients (weighted mean age $=64.4$ years, $59 \%$ females, weighted mean BMI $=29.0 \mathrm{~kg} / \mathrm{m}^{2}$ ) and 343 ageing OA patients (weighted mean age $=78.1$ years, $68 \%$ females, weighted mean $B M I=27.3 \mathrm{~kg} / \mathrm{m}^{2}$ )

Conclusion: Gait analysis as a noninvasive aid to clinical assessment could become an important clinical method of functional assessment in knee OA as well as arthritic changes taking place due to age. However, on the basis of the present systematic literature search, the available data concerning validity and reliability are clearly insufficient to consider kinematic parameters as quantifiable outcome measures in OA.

Summary and implications: Our study finding suggests presence of altered gait parameters because of osteoarthritis of knee as well as ageing, which was supported by result of having higher grading of affection in relatively younger individual have shown less affection because of high functionality level, while in compare to that older individuals less arthritic changes have also shown more impairment in functionality because of low occupational needs.

KEY WORDS: Knee Osteoarthritis, Ageing Individuals, Gait, Exercise Therapy.

Address for correspondence: Dr. Ranveer Kumar Mahato(P.T.), Ph.D. Scholar, Gujarat University, Ahmedabad, Principal, Pioneer Physiotherapy College, Ajwa-Nimeta Road, Sayajipura, Vadodara390019, Gujarat, India. Mo:+919723617191 E-Mail: drranveermahato@gmail.com

Access this Article online

Quick Response code

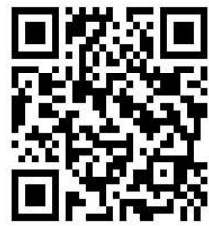

DOI: 10.16965/ijpr.2019.194

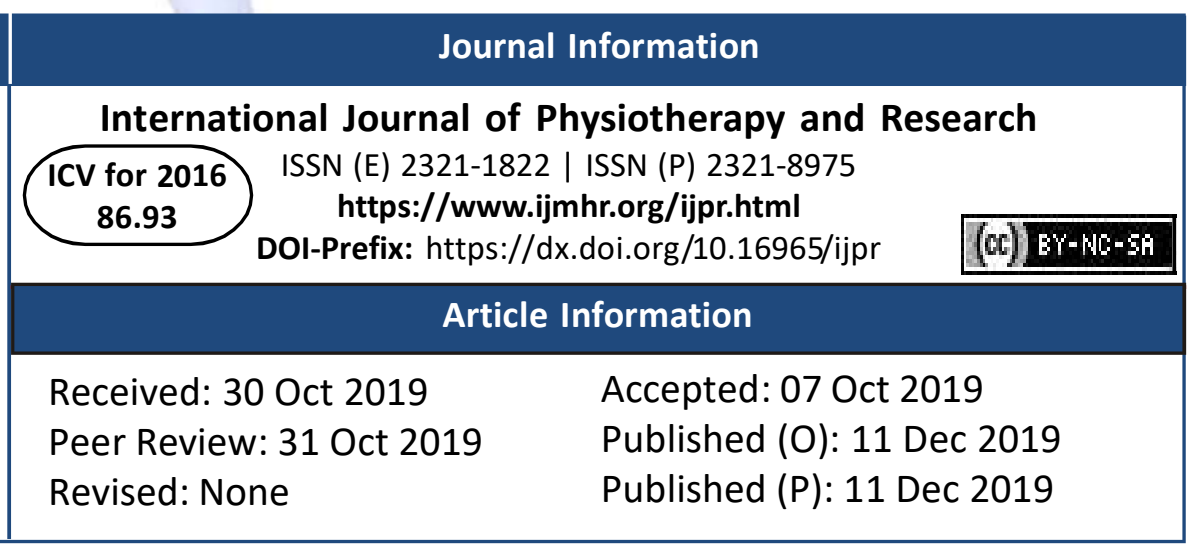




\section{INTRODUCTION}

Health care provision are facing additional burden from ageing adults because osteoarthritis is the most prevalent condition affecting synovial joints leading to substantial pain and disability which is most commonly present in ageing adults [1]. Prevalence increases with age $[2,3]$. In recent treatment guidelines for OA of the knee $[4,5]$, exercise therapy is considered to be an important no pharmacologic treatment approach. The goal of exercise therapy in OA patients is to reduce pain and disability. In order to achieve this, exercise therapy aims at the improvement of muscle strength, stability of joints, range of motion, and aerobic fitness. These functions are frequently impaired in patients with $\mathrm{OA}$, presumably contributing to pain and disability [6]. Improving these functions is assumed to result in a reduction of pain and disability. In addition, exercise therapy aims directly at reduction of disability, e.g., through corrections of the walking pattern [7].

Various articles have been published including previous studies, but later on several new studies on exercise therapy in OA patients have been published [8-12]. These newly published studies were not included in previous reviews [7,13-19], so need arises to include new articles. Furthermore, the methodology for reviews of the literature has evolved considerably [20-22]. Current methodology requires several explicit and systematic steps to be made in conducting reviews of the literature. These steps are a systematic search of the literature [23], selection of studies based on explicit inclusion and exclusion criteria, assessment of methodologic quality [24], and systematic approach toward data extraction and data analysis. Neither the treatment guidelines nor the previously published reviews satisfy the current methodologic requirements for literature reviews. Therefore, considerable improvement can be made by summarizing the evidence available on exercise therapy in OA of the knee by 1) including newly published articles, and 2) applying presently required review methodology. Our objective was to determine the effectiveness of exercise therapy in patients with OA of the knee, based on a systematic review of the evidence from various articles. We focused on the effects of knee osteoarthritis on gait, self-reported disability, observed disability, and patient's global assessment of effect. These outcome measures are recommended fo articles that involve patients with OA of the knee [25].

\section{METHODS}

Literature searchs

Data selection process: A systematic literature search was performed using PUBMED, CINAHL, EBSCO and Willes library databases. Reference sections of the papers initially detected were further searched manually to identify additional relevant reports. Among the resulting abstracts, only articles dealing with knee $\mathrm{OA}$ and reporting at least one of the criteria for gait analysis were selected (feasibility, reliability, validity and responsiveness). As well as articles including any of criteria for ageing individuals were also included. The search was conducted using the following combination of keywords: knee osteoarthritis, gait analysis, ageing individuals, motion gait, kinematic gait or walking analysis. Restrictions were languages other than English. Electromyography or kinetic studies, reports of ambulatory or non-optoelectronic gait systems, review articles and editorials were excluded.

Data extraction: A predetermined grid was used to collect data for each selected article based on reviewing the full text. Information systematically extracted the following information from the included studies: localization (knee), study design, baseline characteristics of patients (age, sex, BMI, occupation, other co-morbid condition), X-ray grades (by Kellgren and Lawrence grading scale). Because of the large number of available kinematic parameters, only the following describers of gait (stride length, gait speed, joint flexion and extension) were studied. Accuracy of data and study methodology were also included in article review procedure.

\section{Assessment of psychometric properties}

Feasibility: It was anticipated that no statistics would be available in the selected reports to evaluate feasibility. Data about equipment cost, procedure duration, apparatus availability and safety were collected.

\section{Validity}

Construct validity: Construct validity was assessed by correlations between kinematic 
parameters and validated $O A$ outcome measures.

Discriminant capacity: Discriminant capacity was assessed by comparing the kinematic parameters of OA and comparators (healthy subjects) in cross-sectional studies by studying given methodology and result databases.

Responsiveness: In longitudinal trials (controlled or not) evaluating OA treatments responsiveness was assessed as change in kinematic parameters after treatment at different time points ( $<3$ months, 3-6 months, $>6$ months).

Flow chart: Flow of articles recruited for the systematic review.

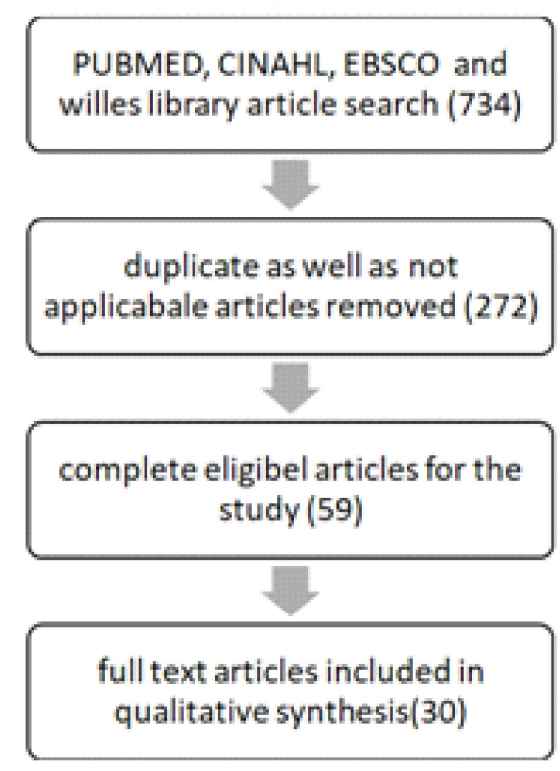

\section{RESULTS}

Data selection process: Among the 272 articles identified by the literature search, 59 articles reporting one of filter criteria were selected. Three additional articles were found by manual search. After reviewing the full-texts, another 29 reports were excluded because preselected 3D kinematic data were not available. Thus, the final review included 30 full-length reports (19 knee OA studies, 11 OA studies including ageing individuals) studying 781 knee OA patients (weighted mean age $=64.4$ years, $59 \%$ females, weighted mean $\mathrm{BMI}=29.0 \mathrm{~kg} / \mathrm{m2}$ ) and 343 ageing OA patients (weighted mean age $=78.1$ years, $68 \%$ females, weighted mean $\mathrm{BMI}=27.3 \mathrm{~kg} / \mathrm{m} 2$ )

Discriminant capacity: Eighteen studies reported data for discriminant capacity, with very similar results in ageing (126 patients) and knee OA (598 patients). Results from 16 studies comparing gait speed and stride length in knee [14-24] and ageing population [25-31]. OA patients with those in healthy subjects indicated a good discriminant capacity, with markedly reduced gait speed (mean relative reduction 13 to $14 \%$ ) and reduced stride length (mean relative reduction $8 \%$ ). In knee OA patients (six studies), knee flexion during walking was decreased in patients compared to healthy subjects (mean relative reduction $18 \%$ ) [14-16,20,32,33]. In elderly OA patients (three studies), conflicting results were found for hip flexion but hip extension was also significantly reduced $[25,27,28]$.

Responsiveness: Six studies [13,19,20,34,35] evaluating knee OA patients reported results concerning responsiveness.

\section{DISCUSSION}

The major increase in the use of 3D gait analysis during the past decade has followed the technological advances in collecting gait parameters. Kinematic data will allow physicians to obtain and process accurate objective measurements of sophisticated movement such as human walking. Henceforth, one of the main challenges today is no longer how to measure data that quantifies human walking but how to use this information so that it can be of clinical benefit. There is still considerable controversy concerning the use of motion analysis as a tool for clinical decisionmaking in $O A$, including the difficulty of interpreting large amounts of information [24] and the limited of validation to date. Indeed, in this systematic literature search, few data were available concerning validity of articles properties in particular reliability and construct validity. On the other hand, discriminant capacity (difference between OA patients and healthy subjects) is demonstrated and responsiveness seems to be satisfactory.

In the present study, similar results have been found between ageing and knee patients for all psychometric properties of kinematic parameters. Therefore, this technique could be applied in both diseases. 3D gait analysis presents feasibility issues, with long procedure 
and expensive systems. Kinematic analysis takes around 30 minutes per patient in our experience, and a vast room dedicated to gait examination is needed. These could be major drawbacks for its use in clinical practice in $O A$ patients. Whereas the reliability of measurement parameters using 3D motion analysis systems has been well evaluated in normal adult gait [34,35], it has not yet been sufficiently evaluated for quantitative kinematic gait variables in OA patients. It is therefore necessary to improve the intersession and intra-session reliability of gait parameters to minimize the numbers of trials and to limit the number of patients to be included in each study.

Concerning construct validity, unexpected weak correlations were found in one study [12] between functional sub scores and selected gait parameters in ageing OA patients. This might suggest that the self-perceived physical status did not reflect objective physical performance such as gait speed. The present results show that 3D gait analysis is capable of discriminating between healthy subjects and OA patients. In a future study, it would be interesting to evaluate the capacity of these parameters to discriminate between $\mathrm{OA}$ of varying severity. Moreover, the results suggest that kinematic parameters are probably responsive outcome measures in OA However, the sensitivity to change remains to be confirmed in additional studies because of conflicting results observed for total joint replacement and because of the lack of sufficient data.

The findings from this study must be considered in the light of its limitations, which reflect limits in the field of motion analysis. Firstly, heterogeneity in gait analysis systems and in experimental walking protocols was noticed. Secondly, the low number of patients included does not provide sufficient statistical power to detect small differences. Thirdly, the heterogeneity of the ageing or knee OA populations and of study designs made systemic-analysis inappropriate. Data must therefore be interpreted with caution. Moreover, only the psychometric properties of kinematic parameters were assessed in the present study and a specific systematic literature search on kinetic and electromyographic parameters in OA of the lowers limbs is now warranted.

Our study finding suggest findings of having gait affection more marked in older adults, which was supported by result of having higher grading of affection in relatively younger individual have shown less affection because of high functionality level, while in compare to that older individuals with less arthritic changes has also shown more impairment in functionality because of low occupational needs [33-35].

In conclusion, our systemic review finding shows alteration in temporo-spastial parameters of gait which is more provoked because of increasing age. In the future, gait analysis as a noninvasive aid to clinical assessment could become an important clinical method of functional assessment in knee OA as well as arthritic changes taking place due to age. However, on the basis of the present systematic literature search, the available data concerning validity and reliability are clearly insufficient to consider kinematic parameters as quantifiable outcome measures in OA. Further, gait analysis studies evaluating a large number of patients and using filter criteria are needed.

\section{ABBREVIATION}

\section{OA - Osteoarthritis}

BMI - Body Mass Index

NSAIDS - Nonsteroidal Anti-inflammatory Drugs ACKNOWLEDGEMENTS

We wish to thank Dr. Dhara N. Panchal for the help in revising the manuscript for her assistance with the systematic literature search.

\section{Conflicts of interest: None}

\section{REFERENCES}

[1]. Wilson MG, Michet CJ, Illstrup DM, Melton L. Idiopathic symptomatic osteoarthritis of the hip and knee; a population based incidence study. Mayo ClinProc 1990;65:1214-21.

[2]. Felson DT, Naimark A, Anderson J, Kazis L, Castelli W, Meenan RF. The prevalence of knee osteoarthritis in the elderly: The Framingham Osteoarthritis Study. Arthritis Rheum 1987;30: 914-8.

[3]. Miedema H. Reuma-onderzoekmeerdere echelons (ROME): basisrapport. Leiden (The Netherlands): NIPG-TNO; 1994.

[4]. Hochberg MC, Altman RD, Brandt KD, Clark BM, Dieppe PA, Griffin MR, et al. Guidelines for the medical management of osteoarthritis. Part I. Osteoarthritis of the knee. Arthritis Rheum 1995;38:1535-40. 
[5]. Hochberg MC, Altman RD, Brandt KD, Clark BM, Dieppe PA, Griffin MR, et al. Guidelines for the medical management of osteoarthritis. Part II. Osteoarthritis of the knee. Arthritis Rheum 1995;38:1541-6.

[6]. Dekker J, Boot B, van der Woude L, Bijlsma JWJ. Pain and disability in osteoarthritis: a review of biobehavioral mechanisms. J Behav Med 1992;15:189-214.

[7]. Dekker J, Mulder PH, Bijlsma JWJ, Oostendorp RAB. Exercise therapy in patients with rheumatoid arthritis and osteoarthritis: a review. AdvBehav Res Ther 1993;15:211-38.

[8]. Laroche D, Pozzo T, Ornetti P, et al. Effects of loss of metatarsophalangeal joint mobility on gait in rheumatoid arthritis patients. Rheumatology (Oxford, England) 2006;45:435-40.

[9]. Missaoui B, Portero P, Bendaya S, et al. Posture and equilibrium in orthopedic and rheumatologic diseases. NeurophysiolClin 2008;38:447-57.

[10]. Lequesne MG. The algofunctional indices knee osteoarthritis. J Rheumatol 1997;24:779-81.

[11].Boers M, Brooks P, Strand CV, et al. The OMERACT filter for outcome measures in rheumatology. J Rheumatol 1998;25:198-9

[12]. Fransen M, Crosbie J, Edmonds J. Reliability of gait measurements in people with osteoarthritis of the knee. PhysTher 1997;77:944-53.

[13].Rudolph KS, Schmitt LC, Lewek MD. Age-related changes in strength, joint laxity, and walking patterns: are they related to knee osteoarthritis? PhysTher 2007;87:1422-32.

[14]. Dujardin F, Aucouturier T, Bocquet G, et al. Kinematics of the healthy and arthritic joint during walking. A study of 136 subjects. Rev ChirOrthop ReparatriceAppar Mot 1998;84:689-99.

[15]. Blin O, Pailhous J, Lafforgue P, et al. Quantitative analysis of walking in patients with knee osteoarthritis: a method of assessing the effectiveness of nonsteroidal anti-inflammatory treatment. Ann Rheum Dis 1990;49:990-3.

[16]. Cheing GL, Hui-Chan CW. The motor dysfunction of patients with knee osteoarthritis in a Chinese population. Arthritis Rheum 2001;45:62-8.

[17]. Kaufman KR, Hughes C, Morrey BF, et al. Gait characteristics of patients with knee osteoarthritis. J Biomech 2001;34:907-15.

[18]. Al-Zahrani KS, Bakheit AM. A study of the gait characteristics of patients with chronic osteoarthritis of the knee. DisabilRehabil 2002;24:275-80.

[19]. Gok H, Ergin S, Yavuzer G. Kinetic and kinematic characteristics of gait in patients with medial knee arthrosis. ActaOrthopScand 2002;73:647-52.

[20]. Chen CP, Chen MJ, Pei YC, et al. Sagittal plane loading response during gait in different age groups and in people with knee osteoarthritis. Am J Phys Med Rehabil 2003;82:307-12.

[21]. Solak AS, Kentel B, Ates Y. Does bilateral total knee arthroplasty affect gait in women? comparison of gait analyses before and after total knee arthroplasty compared with normal knees. J Arthroplasty 2005;20:745-50.
[22]. Mundermann A, Dyrby CO, Andriacchi TP. Secondary gait changes in patients with medial compartment knee osteoarthritis: increased load at the ankle, knee, during walking. Arthritis Rheum 2005;52:2835-44.

[23]. Shakoor N, Block JA.Walking barefoot decreases loading on the lower extremity joints in knee osteoarthritis. Arthritis Rheum 2006;54:2923-7.

[24]. McKean KA, Landry SC, Hubley-Kozey CL, et al. Gender differences exist in osteoarthritic gait. ClinBiomech (Bristol, Avon) 2007;22:400-9.

[25]. Deluzio KJ, Astephen JL. Biomechanical features of gait waveform data associated with knee osteoarthritis: an application of principal component analysis. Gait Posture 2007;25:86-93.

[26]. Hulet C, Hurwitz DE, Andriacchi TP, et al. [Functional gait adaptations in patients with painful knee]. Rev ChirOrthopReparatriceAppar Mot 2000;86:581-9.

[27]. Watelain E, Dujardin F, Babier F, et al. Pelvic and lower limb compensatory actions of subjects in an early stage of knee osteoarthritis. Arch Phys Med Rehabil 2001;82:1705-11.

[28]. Maly MR, Costigan PA, Olney SJ. Role of knee kinematics and kinetics on performance and disability in people with medial compartment knee osteoarthritis. ClinBiomech (Bristol, Avon) 2006;21:10519.

[29]. Bejek Z, Paroczai R, Illyes A, et al. The influence of walking speed on gait parameters in healthy people and in patients with osteoarthritis. Knee Surg Sports TraumatolArthrosc 2006;14:612-22.

[30]. Parent E, Moffet $\mathrm{H}$. Comparative responsiveness of locomotor tests and questionnaires used to follow early recovery after total knee arthroplasty. Arch Phys Med Rehabil 2002;83:70-80.

[31]. Detrembleur C, De Nayer J, van den Hecke A. Celecoxib improves the efficiency of the locomotor mechanism in patients with knee osteoarthritis. A randomised, placebo, double-blind and cross-over trial. Osteoarthritis Cartilage /OARS, Osteoarthritis Res Soc 2005;13:206-10.

[32]. Borjesson M, Weidenhielm L, Mattsson E, et al. Gait and clinical measurements in patients with knee osteoarthritis after surgery: a prospective 5-year followup study. Knee 2005;12:121-7.

[33]. Kadaba M, Ramakrishnan H, Wotten $M$, et al. Repetability of kinematic, kinetic and electromyographic data in normal adult gait. J Orthop Res 1989; 7: 849-60.

[34]. Barker S, Craik R, Freedman W, et al. Accuracy, reliability, and validity of a spatiotemporal gait analysis system. Med EngPhys 2006;28:460-7.

[35]. Davis AM, Perrucio AV, Canizares M, et al. Comparative validity and responsiveness of the HOOS-PS and the KOOS-PS to the WOMAC physical function subscale in total joint replacement for osteoarthritis. Osteoarthritis Cartilage /OARS, Osteoarthritis Res Soc 2009;17:843-7. 